\title{
Percutaneous Transpedicular Interbody Fusion Technique in Percutaneous Pedicle Screw Stabilization for Pseudoarthrosis Following Pyogenic Spondylitis
}

\author{
Ko Ikuta $^{1}$, Keigo Masuda ${ }^{1}$, Yutaka Yonekura ${ }^{2}$, Takahiro Kitamura ${ }^{1}$, Hideyuki Senba ${ }^{1}$, Satoshi Shidahara ${ }^{1}$ \\ ${ }^{1}$ Department of Orthopedic Surgery, Karatsu Red Cross Hospital, Karatsu, Japan \\ ${ }^{2}$ Department of Orthopedic Surgery, Saiseikai Karatsu Hospital, Karatsu, Japan
}

This report introduces a percutaneous transpedicular interbody fusion (PTPIF) technique in posterior stabilization using percutaneous pedicle screws (PPSs). An 81-year-old man presented with pseudoarthrosis following pyogenic spondylitis 15 months before. Although no relapse of infection was found, he complained of obstinate low back pain and mild neurological symptoms. Radiological evaluations showed a pseudoarthrosis following pyogenic spondylitis at T11-12. Posterior stabilization using PPSs from Th9 to L2 and concomitant PTPIF using autologous iliac bone graft at T11-12 were performed. Low back pain and neurological symptoms were immediately improved after surgery. A solid interbody fusion at T11-12 was completed 9 months after surgery. The patient had no restriction of daily activity and could play golf at one year after surgery. PTPIF might be a useful option for perform segmental fusion in posterior stabilization using PPSs.

Keywords: Minimally invasive surgery Procedures; Pseudoarthrosis; Percutaneous; Spinal fusion

\section{Introduction}

Several minimally invasive spine surgeries have been developed over the past 25 years. These include the recently developed percutaneous insertion of pedicle screws as a minimally invasive alternative to the open technique during posterior instrumented fusion procedures [1]. Stabilization using percutaneous pedicle screws (PPSs) can reduce blood loss and postoperative pain, and speed the return to previous activities. The procedure is currently used for several spinal disorders including degenerative disease [2], deformity [3,4], trauma [5,6], infection [7], and metastatic tumor [8]. Good clinical outcomes have been reported. However, the technical drawbacks of the percutaneous procedure include difficulty in bone grafting.

This paper describes the use of percutaneous transpedicular interbody fusion (PTPIF) in posterior stabilization using PPSs. A case report of a patient of pseudoarthrosis following pyogenic spondylitis is presented.

\section{Technical Note}

An 81-year-old man patient suffered from pyogenic spondylitis at T11-12 15-months before the first visit to our

Received Jun 22, 2015; Revised Jul 25, 2015; Accepted Aug 14, 2015

Corresponding author: Ko Ikuta

Department of Orthopedic Surgery, Karatsu Red Cross Hospital, 1-5-1 Futago, Karatsu-city, Saga 847-8588, Japan

Tel: +81-955-72-5111, Fax: +81-955-73-9530, E-mail: kou-ikuta@karatsu.jrc.or.jp 
hospital (Fig. 1). The lesion was successfully treated by conservative treatment including select antibiotic therapy, percutaneous drainage and external immobilization. Although the inflammation was soothed by the conservative treatments for 3 months, obstinate low back pain and mild legs paresthesia persisted. Low back pain was worsen when turning over in bed, getting out of bed or a chair and sitting for a long time.

Physical examination demonstrated tenderness over the thoracolumbar junction. Neurologic examination revealed an absence of the deep tendon reflex of Achilles tendon, hypesthesia on both great toes and mild muscle weakness of toe extensors on both legs (manual muscle testing: grade 4 of 5). Radiographs and computed tomography (CT) scan showed a wedged-deformed T12 vertebra caused by massive bone destruction and vacuum cleft in the T11-12 intervertebral disc surrounded by sclerotic bone edge following T11-12 pyogenic spondylitis (Figs. 2A, B, 3B, D). Diffuse idiopathic skeletal hyperosteosis was concomitantly found. The kyphotic angle of T11-12
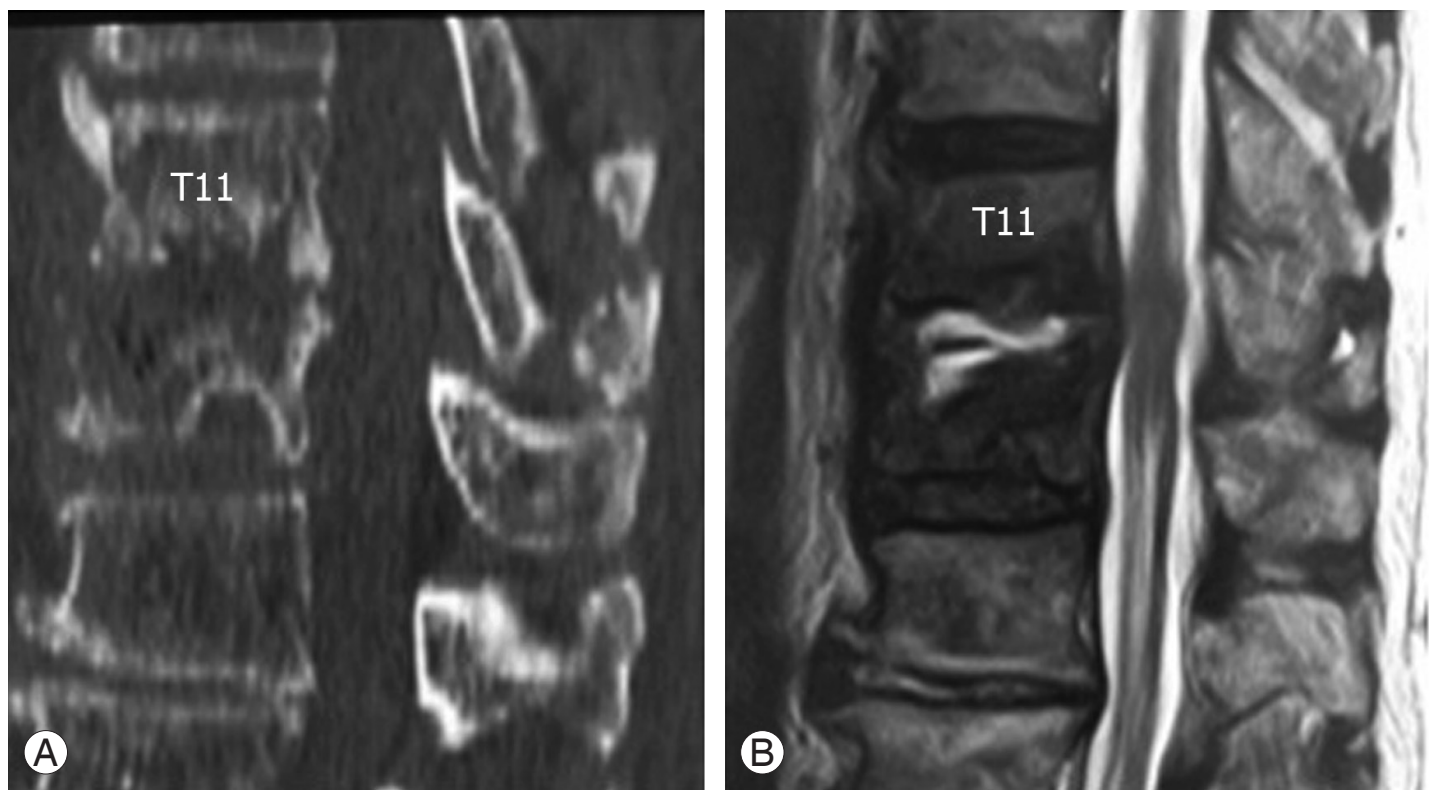

Fig. 1. Computed tomography (CT) and magnetic resonance image (MRI) obtained at 15 months before the first vist to our hospital. (A) Sagittal reformat CT image shows a destructive change at T11-12. (B) Sagittal T2 weighted MRI demonstrates intradiscal abscess formation at T11-12.
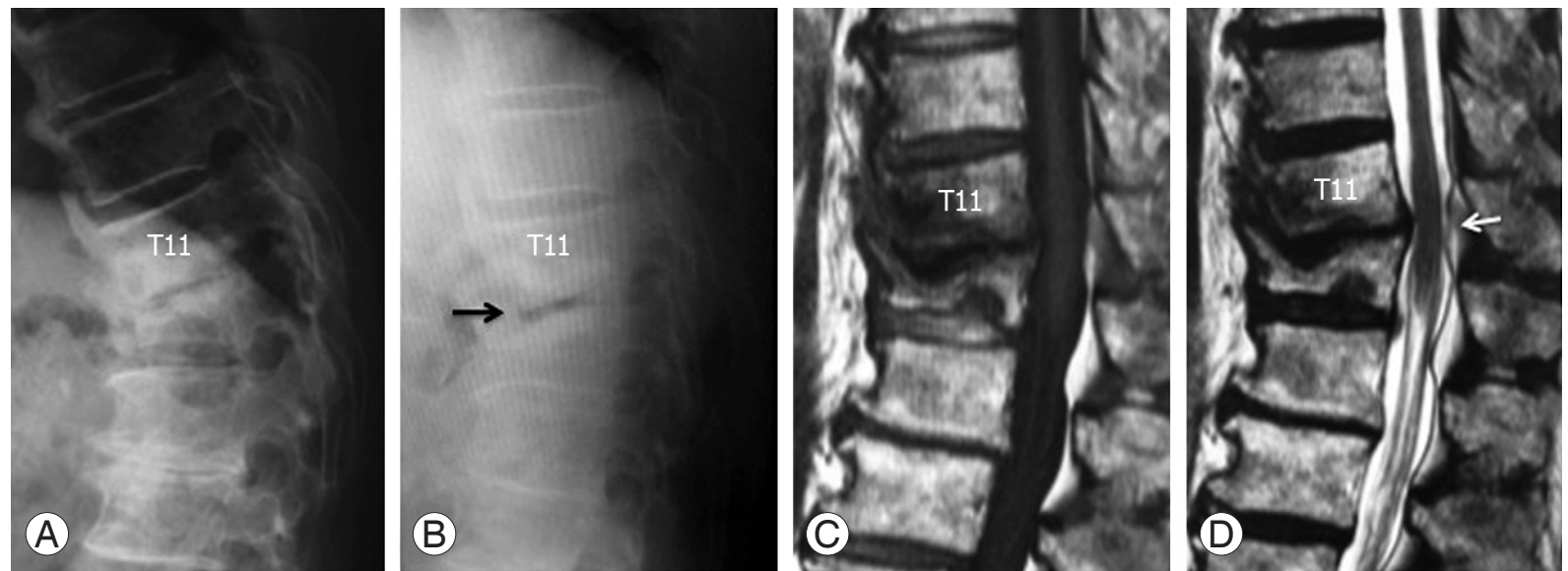

Fig. 2. Lateral radiographs and sagittal magnetic resonance image (MRI) obtained th the first visit to our hospital. (A) Lateral radiograph shows wedged deformed T12 vertebra caused by massive bone destruction following pyogenic spondylitis. (B) Lateral radiograph on supine position demonstrates intradiscal vacuum cleft (black arrow). (C, D) Sagittal T1 weighted (C) and T2 weighted (D) MRI reveal no active inflammation signs. Mild stenosis at T11-12 is evident (white arrow). 
was 27 degrees on standing lateral radiograph and 16 degrees on supine position. Magnetic resonance imaging (MRI) demonstrated mild T11-12 stenosis without active inflammation signs of T11 and T12 vertebrae (Fig. 2C, D). Blood chemistry revealed no active inflammation (leukocyte count, 4,330/uL; C-reactive protein, $0.4 \mathrm{mg} / \mathrm{dL}$ ).

\section{Surgical procedure}

Posterior stabilization using PPS was done utilizing the ILLICO Minimally Invasive Surgery System (Alphatec Spine Inc., Carlsbad, CA, USA) from Th9 to L2 and PTPIF using autologous iliac bone graft at T11-12 was performed (Fig. 4A, B). The procedure was performed with the patient in the prone position after induction of general anesthesia. Skin incisions measuring 2 to $3 \mathrm{~cm}$ in length were marked over each chosen pedicle with assistance of two-dimensional fluoroscopic images. The assistance of fluoroscopic images was needed through the procedure.
A Jamshidi cannulated needle was used to perforate each pedicle from T9 to L2. After confirming that the tip of needle was placed correctly, the needle was led into the vertebral body through the pedicle at each vertebra. A guide wire was then inserted through the needle into the vertebral body. The needle was carefully removed leaving the guide wire in place, and then cannulated screws were placed over the guide wire from T9 to L2, excepting T12. At T12, the needle was inserted with cephalad angulation in the sagittal plane to reach the center of the T11-12 disc. After the needle reached to the disc space with a perforation of the upper endplate at T12, the guide wire was inserted through the needle into the disc space. The needle was carefully removed leaving the guide wire in place. A pedicle screw tapper for PPS was used to widen the pathway to the disc space. The disc space was curetted by small discectomy instruments. After sufficient irrigation of the disc space, cancellous bone harvested from the iliac crest was jammed into the disc space through a cylindrical
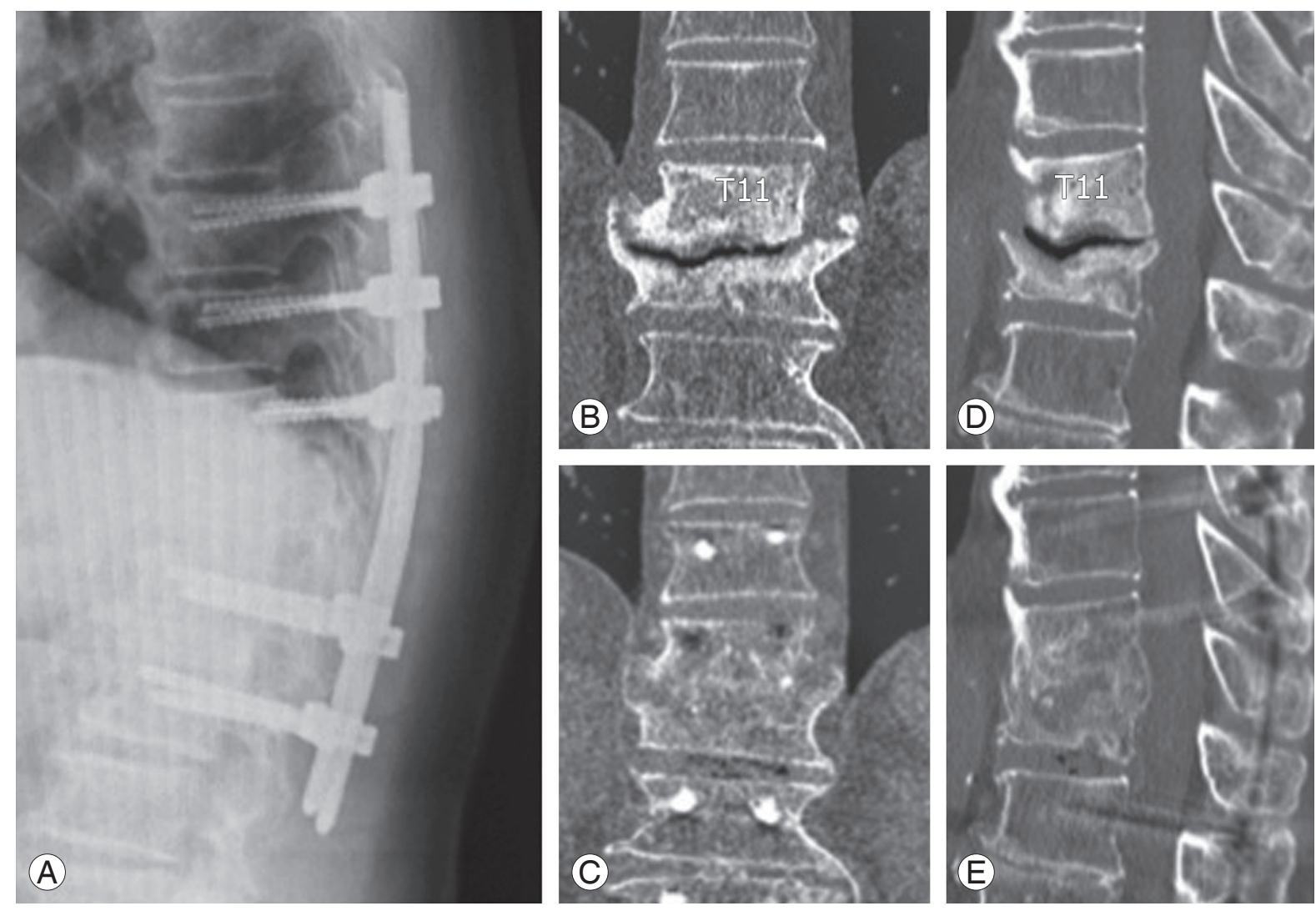

Fig. 3. Lateral radiograph on standing, and preoperative and postoperative reformat computed tomography (CT). (A) Lateral radiograph obtained at one year after surgery demonstrates no correction loss of sagittal alignment. (B, D) Preoperative CT images show pseudoarthrosis with intradiscal vacuum cleft at T11-12. (C, E) CT images obtained at 9 months after surgery demonstrate a completed solid interbody fusion at T11-12. 

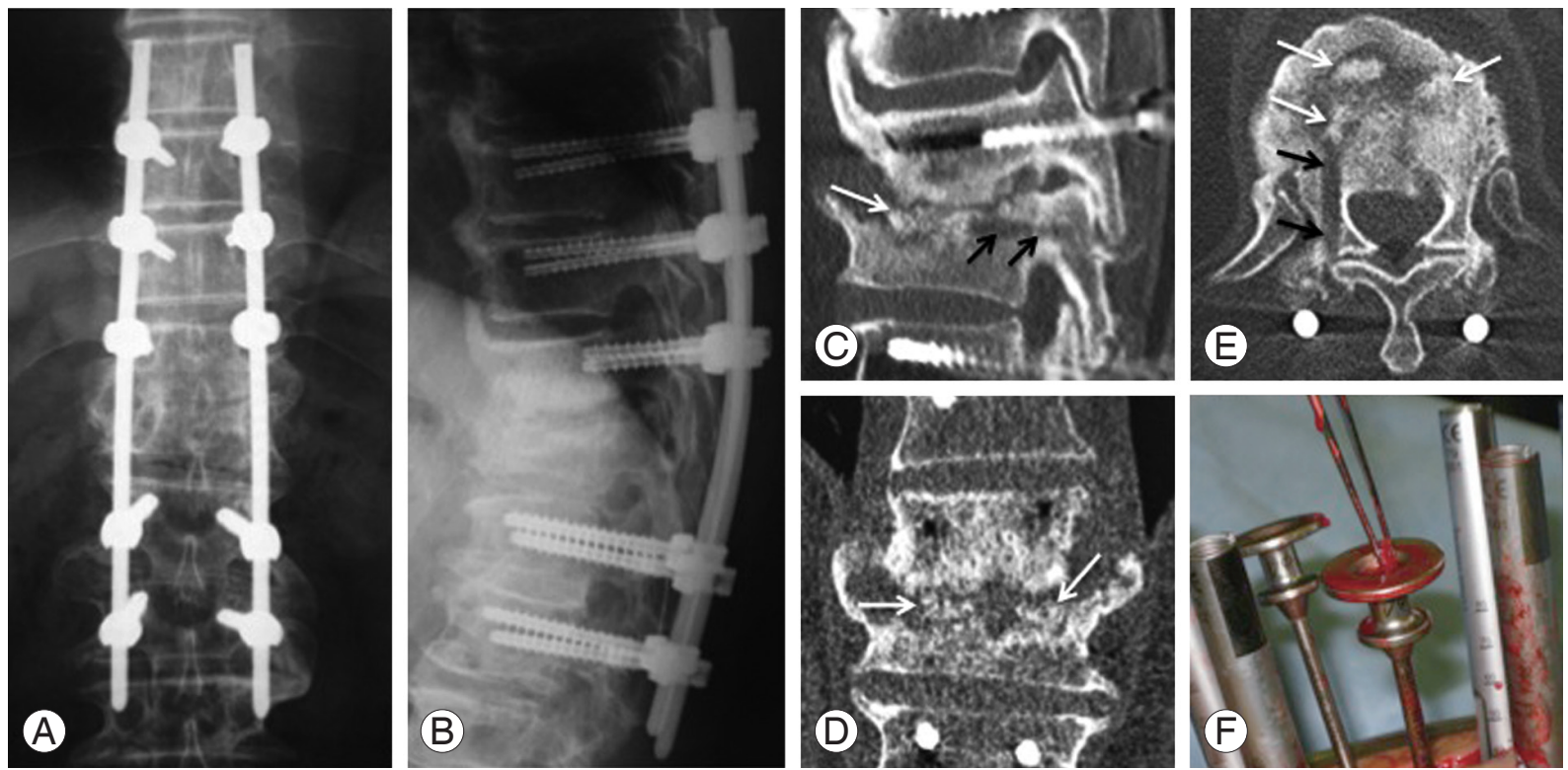

Fig. 4. Postoperative radiographs, computed tomography (CT) and intraoperative photograph. (A, B) Postoperative radiographs. (C-E) Postoperative reformat CT images show a sufficient amount of graft bone in disc space at T11-12 (white arrows) and the pathway route for percutaneous transpedicular interbody fusion in the T12 (black arrows). (F) Intraoperative photograph shows a technique of percutaneous bone graft using cylindrical instrument.

instrument produced for vertebroplasty using hydroxyapatite blocks (Medtronic Inc., Memphis, TN, USA) by a bilateral transpedicular approach. (Fig. 4C-F) Afterwards, the rods of the appropriate bending were placed over the screws through subcutaneous soft tissues and muscles. After any bleeding within the wound was controlled, the surgical incisions were closed.

Operative time was 210 minutes and estimated blood loss during surgery was $95 \mathrm{~mL}$. Postoperative radiograph showed T11-12 kyphotic angle of 16 degrees (Fig. 4B).

\section{Postoperative course}

The patient was mobilized from the day after surgery. Low back pain and neurological symptoms improved immediately after surgery. On postoperative CT evaluation, a sufficient amount of bone graft in T11-12 intervertebral space was revealed (Fig. 4C-E). At 9 months after surgery, a solid interbody fusion at the T11-12 was completed based on assessment using reformat CT images (Fig. 3C, E). A loss of correction on radiographic evaluations was not detected at one year after surgery (T11-12 kyphotic angle: 16 degrees) (Fig. 3A). The patient had no restriction of daily activity and could enjoy playing golf at latest follow-up.

\section{Discussion}

Posterior stabilization using PPSs has been recently developed as a minimally invasive alternative to conventional posterior instrumented fusion procedures [1]. Minimally invasive stabilization is generally applied to several spinal disorders including degenerative disease [2], deformity $[3,4]$, trauma [5,6], infection [7], and metastatic tumor [8], and many authors have described its usefulness for the lesions and good clinical outcomes. Advantages include reduction of blood loss, requirements for postoperative analgesia, reduced length of hospital stay as well as earlier return to previous activity. However, the minimally invasive procedure is not as suited for bone grafting. A minimally invasive surgery grafting technique using a graft packing tube for a posterolateral bone graft has been presented [1]. However, facet joint preparation for bone graft might be complicated and there is concern about the amount of grafting bone.

There is unanimity of opinion that a sufficient amount of bone graft and a good environment of the grafting site are crucial to completing a solid fusion. Compared with the posterolateral technique, interbody fusion has several advantages that include immediate anterior column load shearing, large surface area for fusion, and bone graft subjected to compressive loads. Posterior lumbar interbody 
fusion was originally described in 1953 [9]. The technique has grown in popularity and is now a commonly performed procedure for several degenerative disorders. Transforaminal lumbar imterbody fusion (TLIF) has become increasingly popular as it is a less invasive posterior interbody fusion technique [10]. TLIF has been further developed to a minimally invasive version [11]. However, both procedures for interbody fusion still present percutaneous challenges.

The pedicle is a useful access route to reach the vertebral body and intervertebral disc from the back. Although the assistance of fluoroscopic images is needed in percutaneous techniques, the transpedicular approach is safe and less invasive. With the development and spread of minimally invasive spine surgery, the percutaneous transpedicular approach has been applied to biopsy [12], drainage $[13,14]$, screw insertion, cement augmentation and bone graft to the vertebral body. Although the percutaneous approach is generally used to reach the vertebral body, some authors have used the approach to reach the adjacent intervertebral disc. In 1998, Hadjipavlou et al. [15] described a percutaneous transpedicular discectomy and drainage with access from adjacent caudal pedicles for pyogenic spondylodiscitis. Lee et al. [16] reported transpediclar curettage and drainage with access from adjacent cranial pedicles for infective spondylodiscitis. PTPIF presented in this paper is a modified Hadjipavlou's technique for interbody fusion. The technique allows percutaneous interbody fusion. However, there are several requirements to ensure safety and effectiveness of the procedure. First, the coronal diameters of targeted pedicle have to be an appropriate size to permit curettage of the disc space. Second, the disc height has to be decreased to jam the graft bone into the disc cavity because large pieces of bone cannot be grafted in this technique.

Disadvantages of the percutaneous technique include a lack of strut bone grafting. Therefore, PTPIF cannot be indicated for a patient who needs an anterior spinal column support to treat the lesion. Considering these characteristics of the technique and anatomical structures of each spinal region, the indication of the PTPIF might be limited to lesions in thoracolumbar junction, as in the present case.

The possibility that the present case patient could have been successfully treated by posterior instrumentation alone cannot be denied. However, there was no doubt about an accelerative effect of interbody fusion on the early completion of solid intervertebral fusion. Consequently, the patient could be successfully treated by posterior stabilization using PPSs and concomitant PTPIF, with a good result.

In conclusion, pseudoarthrosis following pyogenic spondylitis at T11-12 could be successfully treated percutaneously by posterior stabilization using PPSs and concomitant PTPIF. The PTPIF technique might be a useful option to perform segmental fusion in posterior stabilization using PPSs.

\section{Conflict of Interest}

No potential conflict of interest relevant to this article was reported.

\section{References}

1. Mobbs RJ, Sivabalan P, Li J. Technique, challenges and indications for percutaneous pedicle screw fixation. J Clin Neurosci 2011;18:741-9.

2. Foley KT, Gupta SK. Percutaneous pedicle screw fixation of the lumbar spine: preliminary clinical results. J Neurosurg 2002;97:7-12.

3. Anand N, Baron EM, Thaiyananthan G, Khalsa K, Goldstein TB. Minimally invasive multilevel percutaneous correction and fusion for adult lumbar degenerative scoliosis: a technique and feasibility study. J Spinal Disord Tech 2008;21:459-67.

4. Wang MY. Improvement of sagittal balance and lumbar lordosis following less invasive adult spinal deformity surgery with expandable cages and percutaneous instrumentation. J Neurosurg Spine 2013;18:4-12.

5. Cimatti M, Forcato S, Polli F, Miscusi M, Frati A, Raco A. Pure percutaneous pedicle screw fixation without arthrodesis of 32 thoraco-lumbar fractures: clinical and radiological outcome with 36-month follow-up. Eur Spine J 2013;22 Suppl 6:S925-32.

6. Vanek P, Bradac O, Konopkova R, de Lacy P, Lacman J, Benes V. Treatment of thoracolumbar trauma by short-segment percutaneous transpedicular screw instrumentation: prospective comparative study with a minimum 2-year follow-up. J Neurosurg Spine 2014; 20:150-6.

7. Deininger MH, Unfried MI, Vougioukas VI, Hubbe U. Minimally invasive dorsal percutaneous spondylodesis for the treatment of adult pyogenic spondylo- 
discitis. Acta Neurochir (Wien) 2009;151:1451-7.

8. Rao PJ, Thayaparan GK, Fairhall JM, Mobbs RJ. Minimally invasive percutaneous fixation techniques for metastatic spinal disease. Orthop Surg 2014;6:187-95.

9. Cloward RB. The treatment of ruptured lumbar intervertebral discs by vertebral body fusion: I. Indications, operative technique, after care. J Neurosurg 1953;10:154-68.

10. Lowe TG, Tahernia AD, O’Brien MF, Smith DA. Unilateral transforaminal posterior lumbar interbody fusion (TLIF): indications, technique, and 2-year results. J Spinal Disord Tech 2002;15:31-8.

11. Peng CW, Yue WM, Poh SY, Yeo W, Tan SB. Clinical and radiological outcomes of minimally invasive versus open transforaminal lumbar interbody fusion. Spine (Phila Pa 1976) 2009;34:1385-9.

12. Stringham DR, Hadjipavlou A, Dzioba RB, Lander P. Percutaneous transpedicular biopsy of the spine.
Spine (Phila Pa 1976) 1994;19:1985-91.

13. Jeanneret B, Magerl F. Treatment of osteomyelitis of the spine using percutaneous suction/irrigation and percutaneous external spinal fixation. J Spinal Disord 1994;7:185-205.

14. Hadjipavlou AG, Katonis PK, Gaitanis IN, Muffoletto AJ, Tzermiadianos MN, Crow W. Percutaneous transpedicular discectomy and drainage in pyogenic spondylodiscitis. Eur Spine J 2004;13:707-13.

15. Hadjipavlou AG, Crow WN, Borowski A, Mader JT, Adesokan A, Jensen RE. Percutaneous transpedicular discectomy and drainage in pyogenic spondylodiscitis. Am J Orthop (Belle Mead NJ) 1998;27:188-97.

16. Lee BH, Lee HM, Kim TH, et al. Transpedicular curettage and drainage of infective lumbar spondylodiscitis: technique and clinical results. Clin Orthop Surg 2012;4:200-8. 